\title{
Cellular mechanisms of complex I-associated pathology
}

\author{
Andrey Y. Abramov ${ }^{1}$ and Plamena R. Angelova ${ }^{1,2}$ \\ ${ }^{1}$ Department of Clinical and Movement Neurosciences, UCL Queen Square Institute of \\ Neurology, London WC1N 3BG, UK. \\ ${ }^{2}$ Department of Pathophysiology, Sechenov First Moscow State Medical University, 119048 \\ Moscow, Russia
}

Corresponding authors: Prof. Andrey Y. Abramov, a.abramov@ucl.ac.uk; Dr Plamena R. Angelova p.stroh@ucl.ac.uk

\begin{abstract}
Mitochondria control vitally important functions in cells, including energy production, cell signalling and regulation of cell death. Considering this, any alteration in mitochondrial metabolism would lead to cellular dysfunction and development of a disease. Large proportion of disorders associated with mitochondria are induced by mutations or chemical inhibition of the mitochondrial complex I - the entry point to the electron transport chain. Subunits of the enzyme NADH: ubiquinone oxidoreductase, are encoded by both nuclear and mitochondrial DNA and mutations in these genes lead to cardio and muscular pathologies and diseases of the central nervous system. Despite such a clear involvement of complex I deficiency in numerous disorders, the molecular and cellular mechanisms leading to the development of pathology are not very clear. In this review we summarise how lack of activity of complex I could differentially change mitochondrial and cellular functions and how these changes could lead to a pathology, following discrete routes.
\end{abstract}

Keywords: complex I, mitochondria, electron transport chain, bioenergetics, calcium, reactive oxygen species 


\section{Introduction}

Mitochondria are distinguishable from the other numerous cellular organelles by their functional importance, which includes control over energy metabolism, involvement in cell signalling and cell death mechanisms and, not last, by the presence of a separate mitochondrial genome, which consists of multiple copies of circular mitochondrial DNA (mtDNA). Mitochondrial biogenesis is controlled by both: nuclear DNA and mitochondrial genome. Each circle of mtDNA encodes 13 protein subunits of the electron transport chain, including subunits of complex I, III and complex IV.

Despite the fact that mitochondria contain multiple enzymes and exert a variety of functions, most of them are dependent on, or interconnected with, the electron transport chain and mitochondrial membrane potential $(\Delta \Psi \mathrm{m})$, which is controlled by mitochondrial respiration. Four protein complexes located in the inner membrane of mitochondria form the electron transport chain, three of them (complex I, III and IV) using the free energy from redox reactions to translocate protons across the inner mitochondrial membrane to generate $\Delta \Psi \mathrm{m}$, which is then used as a motive force for the F0-F1-ATP synthetase to generate ATP in the process of oxidative phosphorylation (1). Maintenance of transmembrane mitochondrial potential is vitally important for all cells, as complete depolarisation which may be induced by a number of factors, including opening of the permeability transition pore (PTP), lead to the release of pro-apoptotic proteins and is followed by cell death (2). $\Delta \Psi \mathrm{m}$ is also crucial for mitochondrial calcium uptake (3) and generation of reactive oxygen species in physiology and pathology (4, 5). All of this renders the proper function of the mitochondrial electron transport chain to be essential factor for multiple processes in the cell.

The major entry point of mitochondrial respiratory chain is the NADH:ubiquinone oxidoreductase or complex I. This is the only enzyme of ETC which catalyses the oxidation of 
matrix NADH (and transfers $4 \mathrm{H}^{+}$per $\mathrm{NADH}$ ) and is the major donor of electrons for the whole respiratory chain (6). Alternative electron donors - complex II or other ubiquinone-reducing enzymes, such as the electron transfer through the flavoprotein-ubiquinone oxidoreductase (ETF-QO), glycerol-3-phosphate dehydrogenase (GPDH) and dihydroorotate dehydrogenase which enters the ETC at complex III, in the absence of complex I activity have significantly reduced efficiency to the respiratory chain (7).

NADH:ubiquinone reductase reaction is reversible, and thus, in vitro, during reverse electron transfer, complex I can carry electrons upstream from ubiquinol for $\mathrm{NAD}^{+}$reduction at the expense of proton-motive force $(6,8)$. Based on this ability of complex I it was suggested that reverse flux of electrons could be the main trigger for $\operatorname{ROS}$ production in pathology $(8,9)$. Complex I consists of two domains: hydrophilic, with a shifted into the matrix (containing all redox centres of this enzyme) and hydrophobic, located in the membrane $(10,11)$. Moreover, complex I is sensitive to partial oxygen pressure changes and this feature renders it to be one of the major players in the response to hypoxic challenges (12). Seven components of the complex I (MTND1, 2, 3, 4, 4L, 5, 6) are encoded by mtDNA the rest of subunits by nuclear DNA (13).

Complex I is important to both mitochondrial function and cellular homeostasis. Hence complex I deficiency is found in, and potentially underlies the pathology of many mitochondrial disorders $(13,14)$. Although molecular or chemical alteration of the complex I should simply modify, or inhibit the function of ETC, the cellular mechanism of pathologies which lead to various diseases is not very clear. In this review we are discussing the changes in mitochondrial and cellular function, induced by complex I deficiencies.

\section{Mammalian complex I-disease causing mutations}


One third of the mitochondrial diseases are caused by mutations in the nuclear or mitochondrial DNA, encoding subunits of complex I (15). Despite the fact that all of these mutations on molecular level lead to the same endpoint effect- a defective respiration and coupled oxidative phosphorylation, they cause a wide variety of diseases - e.g. Leigh syndrome (16-18), leukoencephalomyelopathy $(19,20)$, cardiomyopathy $(21,22)$, Parkinson's and Alzheimer's diseases (23-26), infantile myoclonic epilepsy $(16,27)$, mitochondrial encephalomyopathy, lactic acidosis and stroke-like episodes syndrome - MELAS $(28,29)$, Leber hereditary optic atrophy $(30,31)$, to name a few. Importantly, chemical inhibition of complex I by various toxins, which specifically inhibit complex I, induce neurodegenerative conditions, including Parkinson's disease $(32,33)$. A strong correlation between the types of mutation with the development of certain type of diseases hasn't been possible, mainly because of the fact that the same point mutation could cause several types of symptomatically clearly distinguishable clinical phenotypes, e.g. replacement of the Arg340 in the MT-ND4 subunit can cause Leigh syndrome in one patient and Leber hereditary optic atrophy in another (11). Furthermore, another major driver of pathogenicity is the copy number of mtDNA, which differs in various patients.

\section{ATP deprivation as a trigger of pathogenesis in Complex I mutations}

Mutations of mtDNA or nuclear DNA which encode complex I are associated with a wide spectrum of pathologies, primarily affecting CNS and muscle function - tissues and cells with highest energy demand. However, a number of studies suggested that the main trigger for cell death in complex I-related pathology is not ATP deprivation (34). One of the possible explanations of this could be that the type of cellular models which investigators used for their studies - are mostly easily accessible human cell lines, such as lymphocytes or fibroblasts which have a significantly lower energy consumption rate than neurons or cardiomyocytes. Thus, metabolic signatures of the fibroblasts from patients with MELAS/ Leigh overlap 
syndrome are indistinguishable, but iPSC generated from these fibroblasts show significant difference in their levels of pyruvate, malic acid, palmitic acid, stearic acid, and lactic acid. Interestingly this metabolomic signature was only seen in the undifferentiated state of iPSCs and was lost upon differentiation, thus pointing towards another energy demanding process which may enhance pathology (35). Deletion of complex I subunit NDUFS4 significantly modulates cellular differentiation in stem cells (36). Changes in mitochondrial metabolism in the development of progressive supranuclear palsy pathology is crucial for adipocytes formation from mesenchymal stem cells (37) suggesting importance of overall metabolism and ATP in the process of differentiation. It should be noted that in iPSC-derived neuronal MELAS model, neurons remove their complex I in the time of differentiation via PINK1/Parkin related mitophagy (38) that also suggests another spectrum of complex I pathology, characteristic for the time of differentiation (Figure 1).

However, very often even iPSC- or stem cells-derived neurons does not possess all functional activity which is typical for neurons. Mutations in genes affecting complex I are recognised in patients with epilepsy, but the mechanisms which lead to seizure activity and cell death in these mutations remain unclear. Epilepsy due to hyperexcitability is highly energy dependent process (39), but seizure-like activity (as a frequent calcium oscillations) could not be induced in iPSC or stem cells derived neurons or other cell types in the same way as it could be done in primary neuronal cultures due to lower functionality of glutamate receptors. However, this process could be mimicked in fibroblasts by the step-like increase in concentrations of electrogenic calcium ionophores, which lead to oscillatory $\mathrm{Ca}^{2+}$ signals in fibroblasts, or by repeatable release of $\mathrm{Ca}^{2+}$ from caged compound using flash photolysis (40). Importantly, this method indicates that intracellular $\mathrm{Ca}^{2+}$ levels cannot be compensated in complex I mutated fibroblasts during extensive periods of hyperexcitability due to an energetic collapse (cellular ATP depletion) that could not be seen under resting conditions (40). Fumonisin $\mathrm{B}_{1}$, a 
mycotoxin, involved in neurological conditions that specifically inhibits complex I (41), lead to metabolic collapse in low magnesium seizure models and increased percentage of seizureinduced cell death (42). Specific inhibitor of complex I rotenone is also used in combination with astrocyte-targeting drugs to induce seizures (43).

\section{Effect of complex I deficiency on mitochondrial respiration and $\Delta \Psi m$}

Mutations/deficiency in complex I expectably lead to a complete or partial inhibition of the activity of this enzyme, as supported constantly in the literature. Complex I enzymatic activity essays require minimal amount of biological sample and are widely used in experiments with patient's tissue (44). Changes in respiratory activity can also be easily detected with a number of methods including oxygen consumption, measurements in isolated mitochondria or in whole cells of different nature (45-47). Complex I-dependent respiration can be assessed in cells with mitochondrial mutations by measurement of mitochondrial pool of NADH $(48,49)$.

Moreover, activity of mitochondrial respiration and complex I activity not always reflect for mitochondrial depolarisation. Thus, the value of $\Delta \Psi \mathrm{m}$ in cells with complex I mutations is highly dependent on the medium and supplements which were used for cell maintenance (50), thus in the majority of cell lines with complex I deficiency $\Delta \Psi \mathrm{m}$ is decreased $(46,51)$ (Figure 1). Partial inhibition of complex I in neurons with complex I mutations can be overcompensated by reversal of the activity of $\mathrm{F}_{0}-\mathrm{F}_{1}$-ATPase, which pumps $\mathrm{H}^{+}$to restore mitochondrial membrane potential and leads to an increased $\Delta \Psi \mathrm{m}(48)$. However, this process cannot compensate completely due to limited glycolytic activity in neurons. Altered mitochondrial activity and ATP levels, and thus viability of cells with complex I deficiency (Leigh syndrome), could be restored by membrane-permeable pro-drugs for complex II substrate, succinate (52).

\section{Complex I deficiency and ROS production}


High $\Delta \Psi \mathrm{m}$ in neurons with mutated complex I lead to excessive mitochondrial ROS production, an oxidative stress followed by cell death, which could be prevented by the use of antioxidants (48). Although mitochondrial complex I is believed to be one of the producers of superoxide and $\mathrm{H}_{2} \mathrm{O}_{2}$ in mitochondria (53), deficiency in complex I induces massive increase of ROS production, dependent or independent of $\Delta \Psi \mathrm{m}$ (54) (Scheme 1). One of the possible explanation of this is the reverse electron transfer from complex II to inactive or impaired complex I and it is shown to be more prominent in neurons than fibroblasts or astrocytes ( 9 , 55). In cardiac cells, ischaemic accumulation of succinate enhances reperfusion injury by reverse electron transfer to inhibited complex I (56). However, antioxidant strategy was not confirmed to be protective in patients with complex I deficiency.

\section{Complex I deficiency and mitochondrial calcium}

Mitochondrial membrane potential controls mitochondrial calcium uptake, and $\mathrm{Ca}^{2+}$ is activator of mitochondrial dehydrogenases including the NADH dehydrogenase of complex I $(3,57)$. Lower $\Delta \Psi \mathrm{m}$ in cells with complex I mutations significantly reduces mitochondrial calcium uptake in various cellular models (49). On the same time complex I and II are shown to be important for mitochondrial calcium homeostasis (58). However, specific reduction in mitochondrial $\mathrm{Ca}^{2+}$ transients has been shown to stimulate autophagy in human fibroblasts with complex I mutations (59). Respiratory complex I dysfunction due to mitochondrial DNA mutations shifts the voltage threshold for opening of the permeability transition pore towards resting levels which then also become reduced by lower mitochondrial calcium in these cells (60) (Scheme 1).

\section{Role of complex I dysfunction in Parkinson's disease}

Featuring role for mitochondria, and in particular, the involvement of complex I in the development of Parkinson's disease has been observed in different cellular models, while it has 
been confirmed on the basis of effects of mitochondrial toxins and complex I mutations (26). Histopathological characteristic of Parkinson's disease is the occurrence of Lewy bodies, which is formed by the misfolded protein $\alpha$-synuclein, which is toxic in oligomeric form. Interestingly, several groups demonstrated that oligomeric $\alpha$-synuclein is inhibiting complex I (61-63). Importantly, application of oligomeric $\alpha$-synuclein to neurons with complex I mutation did not show any further inhibition of mitochondrial function in contrast to control and did not enhance cell death that suggest importance of complex I in $\alpha$-synuclein pathology in Parkinson's disease (25).

\section{Perspectives}

i) Field importance Complex I deficiency, whether genetic or acquired, can cause a range of diseases. Understanding how complex I deficiency damages cells could lead to the development of new therapeutic strategies.

ii) Summary of current thinking Recent progress in iPSC and stem cell technology has been proven helpful to the development of cellular models of human tissue from patients. It significantly improved our understanding of why complex I mutations induce pathology in specific tissues. This cellular models are based mainly on either the energy collapse and the oxidative stress features of complex I pathology.

iii) Future directions Further progress in this area could be expected using better cellular and animal models in a near future. A novel knowledge which could complete our understanding of complex I would be the unravelling of the exact function of the complex I subunits which up to now remain unknown. 


\section{References}

1. Mitchell P. Chemiosmotic coupling in oxidative and photosynthetic phosphorylation. 1966. Biochim Biophys Acta. 2011;1807(12):1507-38.

2. Orrenius S, Gogvadze V, Zhivotovsky B. Calcium and mitochondria in the regulation of cell death. Biochem Biophys Res Commun. 2015;460(1):72-81.

3. Abeti $\mathrm{R}$, Abramov AY. Mitochondrial $\mathrm{Ca}(2+)$ in neurodegenerative disorders. Pharmacol Res. 2015;99:377-81.

4. Votyakova TV, Reynolds IJ. DeltaPsi(m)-Dependent and -independent production of reactive oxygen species by rat brain mitochondria. J Neurochem. 2001;79(2):266-77.

5. Angelova PR, Abramov AY. Functional role of mitochondrial reactive oxygen species in physiology. Free Radic Biol Med. 2016;100:81-5.

6. Galkin A, Moncada S. Modulation of the conformational state of mitochondrial complex I as a target for therapeutic intervention. Interface Focus. 2017;7(2):20160104.

7. Sazanov LA. A giant molecular proton pump: structure and mechanism of respiratory complex I. Nat Rev Mol Cell Biol. 2015;16(6):375-88.

8. Vinogradov AD. Catalytic properties of the mitochondrial NADH-ubiquinone oxidoreductase (complex I) and the pseudo-reversible active/inactive enzyme transition. Biochim Biophys Acta. 1998;1364(2):169-85.

9. Stepanova A, Kahl A, Konrad C, Ten V, Starkov AS, Galkin A. Reverse electron transfer results in a loss of flavin from mitochondrial complex I: Potential mechanism for brain ischemia reperfusion injury. J Cereb Blood Flow Metab. 2017;37(12):3649-58.

10. Fiedorczuk K, Letts JA, Degliesposti G, Kaszuba K, Skehel M, Sazanov LA. Atomic structure of the entire mammalian mitochondrial complex I. Nature. 2016;538(7625):406-10.

11. Fiedorczuk K, Sazanov LA. Mammalian Mitochondrial Complex I Structure and Disease-Causing Mutations. Trends Cell Biol. 2018;28(10):835-67.

12. Galkin A, Abramov AY, Frakich N, Duchen MR, Moncada S. Lack of oxygen deactivates mitochondrial complex I: implications for ischemic injury? J Biol Chem. 2009;284(52):36055-61.

13. Ghezzi D, Zeviani M. Human diseases associated with defects in assembly of OXPHOS complexes. Essays Biochem. 2018;62(3):271-86. 
14. Distelmaier F, Koopman WJ, van den Heuvel LP, Rodenburg RJ, Mayatepek E, Willems PH, et al. Mitochondrial complex I deficiency: from organelle dysfunction to clinical disease. Brain. 2009;132(Pt 4):833-42.

15. Kirby DM, Crawford M, Cleary MA, Dahl HH, Dennett $X$, Thorburn DR. Respiratory chain complex I deficiency: an underdiagnosed energy generation disorder. Neurology. 1999;52(6):1255-64. 16. Bugiani M, Invernizzi F, Alberio S, Briem E, Lamantea E, Carrara F, et al. Clinical and molecular findings in children with complex I deficiency. Biochim Biophys Acta. 2004;1659(2-3):136-47.

17. Marin SE, Mesterman R, Robinson B, Rodenburg RJ, Smeitink J, Tarnopolsky MA. Leigh syndrome associated with mitochondrial complex I deficiency due to novel mutations In NDUFV1 and NDUFS2. Gene. 2013;516(1):162-7.

18. Benit P, Slama A, Cartault F, Giurgea I, Chretien D, Lebon S, et al. Mutant NDUFS3 subunit of mitochondrial complex I causes Leigh syndrome. J Med Genet. 2004;41(1):14-7.

19. Pagniez-Mammeri $H$, Lombes A, Brivet $M$, Ogier-de Baulny $H$, Landrieu $P$, Legrand $A$, et al. Rapid screening for nuclear genes mutations in isolated respiratory chain complex I defects. Mol Genet Metab. 2009;96(4):196-200.

20. Benit P, Chretien D, Kadhom N, de Lonlay-Debeney P, Cormier-Daire V, Cabral A, et al. Largescale deletion and point mutations of the nuclear NDUFV1 and NDUFS1 genes in mitochondrial complex I deficiency. Am J Hum Genet. 2001;68(6):1344-52.

21. Haack TB, Haberberger B, Frisch EM, Wieland T, luso A, Gorza M, et al. Molecular diagnosis in mitochondrial complex I deficiency using exome sequencing. J Med Genet. 2012;49(4):277-83.

22. Haack TB, Madignier F, Herzer M, Lamantea E, Danhauser K, Invernizzi F, et al. Mutation screening of 75 candidate genes in 152 complex I deficiency cases identifies pathogenic variants in 16 genes including NDUFB9. J Med Genet. 2012;49(2):83-9.

23. Nishioka K, Vilarino-Guell C, Cobb SA, Kachergus JM, Ross OA, Hentati E, et al. Genetic variation of the mitochondrial complex I subunit NDUFV2 and Parkinson's disease. Parkinsonism Relat Disord. 2010;16(10):686-7.

24. Hutchin T, Cortopassi G. A mitochondrial DNA clone is associated with increased risk for Alzheimer disease. Proc Natl Acad Sci U S A. 1995;92(15):6892-5.

25. Reeve AK, Ludtmann MH, Angelova PR, Simcox EM, Horrocks MH, Klenerman D, et al. Aggregated alpha-synuclein and complex I deficiency: exploration of their relationship in differentiated neurons. Cell Death Dis. 2015;6:e1820.

26. Schapira AH, Cooper JM, Dexter D, Jenner P, Clark JB, Marsden CD. Mitochondrial complex I deficiency in Parkinson's disease. Lancet. 1989;1(8649):1269. 
27. Schuelke M, Smeitink J, Mariman E, Loeffen J, Plecko B, Trijbels F, et al. Mutant NDUFV1 subunit of mitochondrial complex I causes leukodystrophy and myoclonic epilepsy. Nat Genet. 1999;21(3):260-1.

28. Liolitsa D, Rahman S, Benton S, Carr L, Hanna MG. Is the mitochondrial complex I ND5 gene a hot-spot for MELAS causing mutations? Ann Neurol. 2003;53(1):128-32.

29. Kirby DM, McFarland R, Ohtake A, Dunning C, Ryan MT, Wilson C, et al. Mutations of the mitochondrial ND1 gene as a cause of MELAS. J Med Genet. 2004;41(10):784-9.

30. Yu-Wai-Man P, Chinnery PF. Leber Hereditary Optic Neuropathy. In: Adam MP, Ardinger HH, Pagon RA, Wallace SE, Bean LH, Stephens K, et al., editors. GeneReviews((R)). Seattle (WA)1993.

31. Howell N, Bindoff LA, McCullough DA, Kubacka I, Poulton J, Mackey D, et al. Leber hereditary optic neuropathy: identification of the same mitochondrial ND1 mutation in six pedigrees. Am J Hum Genet. 1991;49(5):939-50.

32. Panov A, Dikalov S, Shalbuyeva N, Taylor G, Sherer T, Greenamyre JT. Rotenone model of Parkinson disease: multiple brain mitochondria dysfunctions after short term systemic rotenone intoxication. J Biol Chem. 2005;280(51):42026-35.

33. Sherer TB, Richardson JR, Testa CM, Seo BB, Panov AV, Yagi T, et al. Mechanism of toxicity of pesticides acting at complex I: relevance to environmental etiologies of Parkinson's disease. J Neurochem. 2007;100(6):1469-79.

34. Watabe M, Nakaki T. ATP depletion does not account for apoptosis induced by inhibition of mitochondrial electron transport chain in human dopaminergic cells. Neuropharmacology. 2007;52(2):536-41.

35. Hattori T, Hamazaki T, Kudo S, Shintaku H. Metabolic Signature of MELAS/Leigh Overlap Syndrome in Patient-specific Induced Pluripotent Stem Cells Model. Osaka City Med J. 2016;62(2):6976.

36. Johnson J, Lee W, Frazier AE, Vaghjiani V, Laskowski A, Rodriguez AL, et al. Deletion of the Complex I Subunit NDUFS4 Adversely Modulates Cellular Differentiation. Stem Cells Dev. 2016;25(3):239-50.

37. Angelova PR, Barilani M, Lovejoy $C$, Dossena $M$, Vigano $M$, Seresini A, et al. Mitochondrial dysfunction in Parkinsonian mesenchymal stem cells impairs differentiation. Redox Biol. 2018;14:47484.

38. Hamalainen RH, Manninen $\mathrm{T}$, Koivumaki H, Kislin M, Otonkoski $\mathrm{T}$, Suomalainen A. Tissue- and cell-type-specific manifestations of heteroplasmic mtDNA 3243A>G mutation in human induced pluripotent stem cell-derived disease model. Proc Natl Acad Sci U S A. 2013;110(38):E3622-30. 
39. Kovac S, Domijan AM, Walker MC, Abramov AY. Prolonged seizure activity impairs mitochondrial bioenergetics and induces cell death. J Cell Sci. 2012;125(Pt 7):1796-806.

40. Kovac S, Preza E, Houlden H, Walker MC, Abramov AY. Impaired Bioenergetics in Mutant Mitochondrial DNA Determines Cell Fate During Seizure-Like Activity. Mol Neurobiol. 2019;56(1):32134.

41. Domijan AM, Abramov AY. Fumonisin B1 inhibits mitochondrial respiration and deregulates calcium homeostasis--implication to mechanism of cell toxicity. Int J Biochem Cell Biol. 2011;43(6):897-904.

42. Domijan AM, Kovac S, Abramov AY. Impact of fumonisin B1 on glutamate toxicity and low magnesium-induced seizure activity in neuronal primary culture. Neuroscience. 2012;202:10-6.

43. Chan F, Lax NZ, Voss CM, Aldana BI, Whyte S, Jenkins A, et al. The role of astrocytes in seizure generation: insights from a novel in vitro seizure model based on mitochondrial dysfunction. Brain. 2019;142(2):391-411.

44. Kanabus M, Fassone E, Hughes SD, Bilooei SF, Rutherford T, Donnell MO, et al. The pleiotropic effects of decanoic acid treatment on mitochondrial function in fibroblasts from patients with complex I deficient Leigh syndrome. J Inherit Metab Dis. 2016;39(3):415-26.

45. Ma H, Folmes CD, Wu J, Morey R, Mora-Castilla S, Ocampo A, et al. Metabolic rescue in pluripotent cells from patients with mtDNA disease. Nature. 2015;524(7564):234-8.

46. McKenzie M, Liolitsa D, Akinshina N, Campanella M, Sisodiya S, Hargreaves I, et al. Mitochondrial ND5 gene variation associated with encephalomyopathy and mitochondrial ATP consumption. J Biol Chem. 2007;282(51):36845-52.

47. Rafikov R, Sun X, Rafikova O, Louise Meadows M, Desai AA, Khalpey Z, et al. Complex I dysfunction underlies the glycolytic switch in pulmonary hypertensive smooth muscle cells. Redox Biol. 2015;6:278-86.

48. Abramov AY, Smulders-Srinivasan TK, Kirby DM, Acin-Perez R, Enriquez JA, Lightowlers RN, et al. Mechanism of neurodegeneration of neurons with mitochondrial DNA mutations. Brain. 2010;133(Pt 3):797-807.

49. McKenzie M, Duchen MR. Impaired Cellular Bioenergetics Causes Mitochondrial Calcium Handling Defects in MT-ND5 Mutant Cybrids. PLoS One. 2016;11(4):e0154371.

50. lannetti EF, Smeitink JAM, Willems P, Beyrath J, Koopman WJH. Rescue from galactoseinduced death of Leigh Syndrome patient cells by pyruvate and NAD(). Cell Death Dis. 2018;9(11):1135. 51. Leipnitz G, Mohsen AW, Karunanidhi A, Seminotti B, Roginskaya VY, Markantone DM, et al. Evaluation of mitochondrial bioenergetics, dynamics, endoplasmic reticulum-mitochondria crosstalk, 
and reactive oxygen species in fibroblasts from patients with complex I deficiency. Sci Rep. 2018;8(1):1165.

52. Ehinger JK, Piel S, Ford R, Karlsson M, Sjovall F, Frostner EA, et al. Cell-permeable succinate prodrugs bypass mitochondrial complex I deficiency. Nat Commun. 2016;7:12317.

53. Angelova PR, Abramov AY. Role of mitochondrial ROS in the brain: from physiology to neurodegeneration. FEBS Lett. 2018;592(5):692-702.

54. Leman G, Gueguen N, Desquiret-Dumas V, Kane MS, Wettervald C, Chupin S, et al. Assembly defects induce oxidative stress in inherited mitochondrial complex I deficiency. Int J Biochem Cell Biol. 2015;65:91-103.

55. Bird MJ, Wijeyeratne XW, Komen JC, Laskowski A, Ryan MT, Thorburn DR, et al. Neuronal and astrocyte dysfunction diverges from embryonic fibroblasts in the Ndufs4fky/fky mouse. Biosci Rep. 2014;34(6):e00151.

56. Chouchani ET, Pell VR, Gaude E, Aksentijevic D, Sundier SY, Robb EL, et al. Ischaemic accumulation of succinate controls reperfusion injury through mitochondrial ROS. Nature. 2014;515(7527):431-5.

57. McCormack JG, Denton RM. The effects of calcium ions and adenine nucleotides on the activity of pig heart 2-oxoglutarate dehydrogenase complex. Biochem J. 1979;180(3):533-44.

58. Jana F, Bustos G, Rivas J, Cruz P, Urra F, Basualto-Alarcon C, et al. Complex I and II are required for normal mitochondrial $\mathrm{Ca}(2+)$ homeostasis. Mitochondrion. 2019;49:73-82.

59. Granatiero V, Giorgio V, Cali T, Patron M, Brini M, Bernardi P, et al. Reduced mitochondrial $\mathrm{Ca}(2+)$ transients stimulate autophagy in human fibroblasts carrying the $13514 \mathrm{~A}>\mathrm{G}$ mutation of the ND5 subunit of NADH dehydrogenase. Cell Death Differ. 2016;23(2):231-41.

60. Porcelli AM, Angelin A, Ghelli A, Mariani E, Martinuzzi A, Carelli V, et al. Respiratory complex I dysfunction due to mitochondrial DNA mutations shifts the voltage threshold for opening of the permeability transition pore toward resting levels. J Biol Chem. 2009;284(4):2045-52.

61. Luth ES, Stavrovskaya IG, Bartels T, Kristal BS, Selkoe DJ. Soluble, prefibrillar alpha-synuclein oligomers promote complex I-dependent, Ca2+-induced mitochondrial dysfunction. J Biol Chem. 2014;289(31):21490-507.

62. Abramov AY, Berezhnov AV, Fedotova El, Zinchenko VP, Dolgacheva LP. Interaction of misfolded proteins and mitochondria in neurodegenerative disorders. Biochem Soc Trans. 2017.

63. Ludtmann MHR, Angelova PR, Horrocks MH, Choi ML, Rodrigues M, Baev AY, et al. alphasynuclein oligomers interact with ATP synthase and open the permeability transition pore in Parkinson's disease. Nat Commun. 2018;9(1):2293. 
Figure 1. Complex I deficiency results in cellular pathology. Complex I alterations/mutations lead to inhibition of respiration, decrease in ATP level, ROS production increase and abnormal metabolism, that lead to lipid peroxidation (LP) and reduction in GSH level. Slower mitochondrial calcium efflux results in pathologically elevated level of $\mathrm{Ca}^{2+}$ in mitochondria, and taken together with all the former events open the mitochondrial permeability transition pore and leads to cell death. 


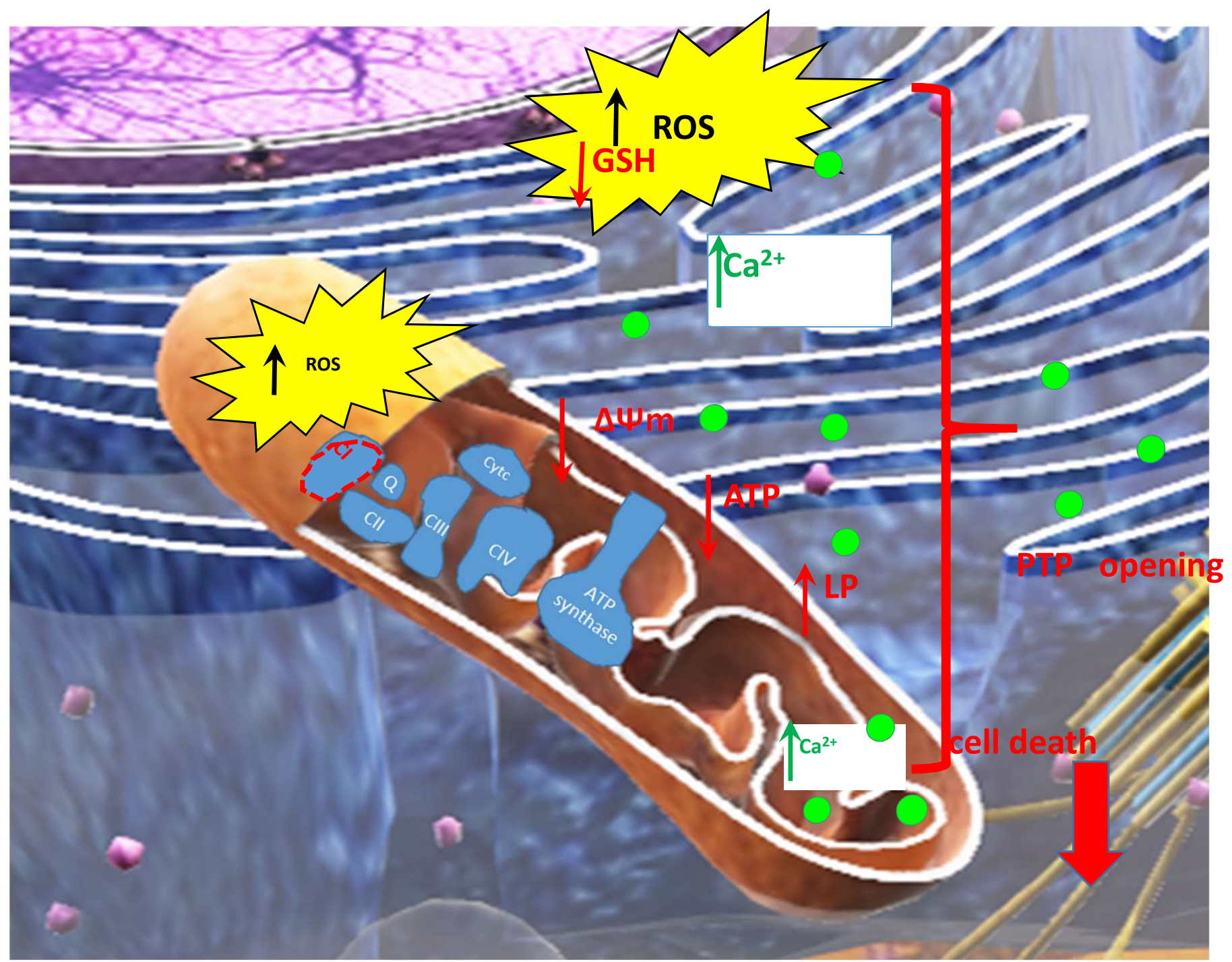

Figure 1 